\title{
Re: Failure of Botulinum Toxin Injection for Neurogenic Detrusor Overactivity: Switch of Toxin Versus Second Injection of the Same Toxin
}

\author{
Benoit Peyronnet1,2, Evelyne Castel-Lacanal3, Andréa Manunta2, Mathieu Roumiguié1, Philippe \\ Marque3, Pascal Rischmann1, Xavier Gamé1
}

${ }^{1}$ Centre Hospitalier Universitaire Rangueil, Department of Urology, Toulouse, France

2 Centre Hospitalier Universitaire Rennes, Department of Urology, Rennes, France

${ }^{3}$ Centre Hospitalier Universitaire Rangueil, Department of Physical Medicine, Toulouse, France

Int J Urol 2015. doi: 10.1111/iju.12950. [Epub ahead of print]

\section{EDITORIAL COMMENT}

Botulinum toxin A (BoNT-A) is the gold standard treatment approach for neurogenic detrussor overactivity (NDO) resistant to anticholinergic treatment. In a study by Peyronnet et al., patients in NDO patient group resistant to anticholinergic treatment with unsuccessful BoNT-A (abobotulinumtoxinA or onabotulinumtoxinA) after the first injection were administered a repeat injection of the same dose of the same toxin in one group, while in the other group, a repeat injection of a different toxin (switch from abobotulinumtoxinA to onabotulinumtoxinA or vice versa) was administered and the results were compared. All patients were evaluated with a 3-day bladder diary and urodynamic testing before treatment and six weeks after treatment. The results of the study showed that the treatment with BoNT-A injection was unsecseful in 60 of 291 patients, (20.6\%). With 58 patients complying with the criteria included in the study, 29 patients had the same dose of the same toxin administered (7 patients abobotulinumtoxinA, 22 patients onabotulinumtoxinA), while the other 29 patients were switched to a different toxin (17 patients switched from abobotulinumtoxinA (Dysport) to onabotulinumtoxinA (Botox), 12 patients switched from onabotulinumtoxinA to abobotulinumtoxinA). The treatment success rate in switched patients was found to be higher (51.7\% vs. $24.1 \%$, $p=0.03)$. Similarly, the recovery of NDO was identified to be higher in the switched patient group (58.6\% vs. 31\%, $p=0.04)$. Also, in the switched patient group, the cystometric capacity increased and maximum detrusor pressure decreased significantly, while there was no change in the group without switched toxins. In the switched patient group, the choice of toxin for first injection did not affect the treatment success. Based on current findings, the researchers concluded that in the NDO patient group with unsuccessful BoNT-A injection, switching the $2^{\text {nd }}$ injection increased treatment success and the choice of toxin for the first injection did not affect switch success. 